\title{
Gesunde Ärztinnen und Ärzte: vom Studium bis zur Pensionierung
}

\section{Christine Romann}

Dr. med., Mitglied des Zentralvorstandes der FMH, Departementsverantwortliche Gesundheitsförderung und Prävention

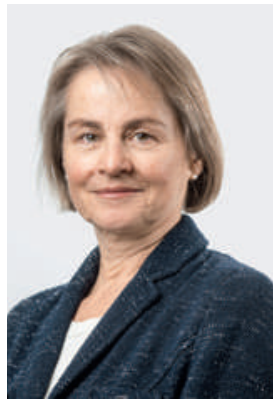

Ärztinnen und Ärzte sind trotz hoher Arbeitsbelastung mehrheitlich zufrieden mit ihrem Beruf. Sie schätzen die vielfältigen Anforderungen ihrer Arbeit, empfinden ihre Tätigkeit als sinnvoll und freuen sich über die Wertschätzung, die sie immer wieder erfahren. Weil sie ihre Arbeit weitgehend autonom und nach eigenem Ermessen gestalten können, sind sie sehr motiviert und leistungsbereit.

Hohe Arbeitslast, lange Arbeitszeiten und die Fokussierung auf die Bedürfnisse der Patientinnen und Patienten bergen aber auch Risiken: Wenn das Privatleben, die sozialen Beziehungen ausserhalb der Berufssphäre und die Regeneration insgesamt zu kurz kommen, drohen dysfunktionale Bewältigungsstrategien und allmähliche Erschöpfung. Der in den letzten Jahren erfolgte Wandel der ärztlichen Arbeitsbedingungen - Autonomieverlust, Zunahme externer Kontrollen sowie berufsfremder Tätigkeiten etc. schränkt zudem den Entscheidungsspielraum und die eigenen Gestaltungsmöglichkeiten ein - mit Folgen für die Berufszufriedenheit.

\section{Obwohl Ärztinnen und Ärzte die Gesundheits- fachpersonen sind, achten sie selbst häufig zu wenig auf ihr eigenes Wohlbefinden.}

Die Gesundheit von Ärztinnen und Ärzten ist ein kostbares Gut, nicht nur für die Betroffenen selbst, sondern auch für die Patienten. Es hat durchaus Auswirkungen auf die Patientenversorgung, wenn Ärzte überlastet und unzufrieden sind: Sie laufen als Behandler häufiger Gefahr, Fehlentscheidungen zu treffen, sind schlechte Rollenvorbilder für ihre Patienten und verursachen nicht zuletzt auch Kosten durch krankheitsbedingte Ausfälle. Sorge tragen für die eigene Gesundheit ist daher nicht nur ein Gebot der Selbstfürsorge von Ärztinnen und Ärzten, sondern auch der Fürsorgepflicht aller, die für deren Arbeitsbedingungen verantwortlich sind. Nicht zuletzt ist es auch eine gute Investition in die Qualität der Gesundheitsvorsorge.

Obwohl Ärztinnen die eigentlichen Experten für Gesundheit sind, lässt ihr Umgang mit der eigenen
Gesundheit mitunter zu wünschen übrig: Viele bekunden Mühe, adäquat Hilfe zu suchen und sich betreuen zu lassen, und nur eine Minderheit hat einen eigenen Hausarzt. Ärzte verschreiben sich Medikamente häufig selber - nicht nur bei Bagatellerkrankungen - und laufen damit Gefahr, ihre Erkrankungen zu unterschätzen oder gar in eine Abhängigkeit abzugleiten.

\section{Das FMH-Symposium vom 7. April 2016 will} Mediziner, Spitäler, Aus- und Weiterbildende für die Ärztegesundheit sensibilisieren.

Die FMH als Dachverband der Schweizer Ärzteschaft nimmt das Thema nun mit dem Symposium «Gesunde Ärzte: vom Studium bis zur Pensionierung» auf, welches am 7. April in Bern stattfindet. Im Zentrum der Veranstaltung geht es dabei um die Frage, wie Ärzte besser auf die eigene Gesundheit achten können, um trotz ihres herausfordernden Berufes nicht auszubrennen. Es gilt, sie selber für ihre eigene Gesundheit zu sensibilisieren, aber auch die Spitäler als Arbeitgeber anzusprechen.

Spannende Referate und Workshops stehen auf dem Programm: Frau Dr. med. Andrée Rochfort, Qualitätsbeauftragte der irischen Grundversorger und Mitglied des WONCA-Vorstandes, berichtet über Erfahrungen aus Irland und England. Als Arbeitspsychologe stellt Prof. Dr. Norbert Semmer das Thema in einen grösseren Zusammenhang und zeigt die Wechselwirkungen zwischen Arbeit und Gesundheit auf - auch in Spitälern. Spannende Workshops entlang der gesamten ärztlichen Berufslaufbahn wie «Survive studies - wie viele Wege führen nach Rom?» oder "Time-out statt Burn-out» laden anschliessend zum Austausch mit Expertinnen und Berufskollegen über bisherige Erfahrungen und mögliche Lösungen ein.

Die Erkenntnisse des Symposiums bilden die Grundlage für das weitere Engagement der FMH in diesem Themenfeld - gestalten Sie dieses mit Ihrer Teilnahme am Symposium mit, Näheres dazu erfahren Sie über www.fmh.ch $\rightarrow$ Politik \& Themen $\rightarrow$ Gesundheitsförderung und Prävention $\rightarrow$ Symposium Gesunde Ärzte! 